\title{
miR-155 inhibition represents a potential valuable regulator in mitigating myocardial hypoxia/reoxygenation injury through targeting BAG5 and MAPK/JNK signaling
}

\author{
$\mathrm{JING} \mathrm{XI}^{1}$, QIANG-QIANG LI ${ }^{2}$, BING-QIANG LI ${ }^{1}$ and NING LI ${ }^{1}$ \\ Departments of ${ }^{1}$ Cardiology and ${ }^{2}$ Cardiology in Integrated Traditional Chinese and Western Medicine, \\ Anqiu People's Hospital, Weifang, Shandong 262100, P.R. China
}

Received May 5, 2019; Accepted September 27, 2019

DOI: $10.3892 / \mathrm{mmr} .2020 .10924$

\begin{abstract}
Increasing evidence has indicated that miR-155 is closely associated with apoptosis, which may protect the myocardium and diminish the infarct area in myocardial ischemia reperfusion injury (IRI). In addition, studies have revealed that miR-155 serves a leading role in promoting fibroblast inflammation, cardiac dysfunction and other aspects of myocardial injury. The present study aimed to uncover the function and potential biological mechanism of miR-155 in myocardial IRI. The rat $\mathrm{H} 9 \mathrm{c} 2$ myocardial cells was treated with hypoxia/reoxygenation $(\mathrm{H} / \mathrm{R})$ to simulate IRI in vitro. Reverse transcription-quantitative polymerase chain reaction (RT-qPCR) was used to detect the expression levels of miR-155 mRNA. Cell Counting Kit- 8 and flow cytometry assays and western blot analysis were applied to determine the biological behaviors of the H/R-treated cells. The association between miR-155 and BAG family molecular chaperone regulator 5 (BAG5) was predicted by bioinformatics software and was confirmed by dual luciferase assay. RT-qPCR and western blot analysis were used to analyze the expression of BAG5. The key proteins involved in mitogen-activated protein kinase (MAPK)/JNK signaling pathway were detected by western blot analysis. The data from the RT-qPCR assay indicated that the expression of miR-155 was markedly upregulated in the H/R model, and that downregulation of miR-155 may promote cell proliferation and inhibit cell apoptosis, and vice versa. BAG5, which was downregulated in the H/R model, was confirmed as a target of miR-155 and negatively modulated by miR-155. The key proteins involved in MAPK/JNK signaling, which were highly expressed in the H/R model, were suppressed by
\end{abstract}

Correspondence to: Mr. Ning Li, Department of Cardiology, Anqiu People's Hospital, 259 Health Road, Anqiu, Weifang, Shandong 262100, P.R. China

E-mail:1n1006@163.com

Key words: myocardial ischemia reperfusion injury, apoptosis, microRNA-155, BAG family molecular chaperone regulator 5, mitogen-activated protein kinase/JNK treatment with the miR-155 inhibitor, and overexpression of BAG5 promoted the protective effect of miR-155 inhibition on cell injury caused by H/R. In addition, the expression patterns of hypoxia-inducible factor 1- $\alpha$ and von Hippel-Lindau were altered following different treatments. Taken together, the data from the present study indicated that miR-155 inhibition represented a potential treatment strategy to improve myocardial H/R injury, which may be associated with targeting BAG5 and inhibition of the MAPK/JNK pathway.

\section{Introduction}

A lack of blood flow into the heart muscle results in a disruption to the supply and demand of oxygen, termed ischemia, which leads to damage or dysfunction of the heart tissue. The subsequent reperfusion that occurs following ischemia can also cause injury. This event is named myocardial ischemia reperfusion injury (IRI). Myocardial IRI, as a common physiological and pathological phenomenon, was first described by Jennings et al (1) in 1960 and often occurs in myocardial infarction and numerous types of cardiac surgeries. In addition, it also causes inflammation, which leads to further harm to the normal tissues around the infarct site. Therefore, myocardial IRI is a major challenge in organ transplantation and surgery (2). Although significant progress has been made in treating ischemia/reperfusion (I/R) mechanisms based on the acute myocardial infarction model, the results of clinical studies have been largely unsatisfactory, which may be due to an inadequate understanding of the mechanisms involved. Hypoxia/reoxygenation (H/R) injury, a mimic in vitro model of myocardial I/R injury, has been widely used to explore the underlying molecular mechanism of myocardial I/R injury (3-5). At present, a number of studies have explored myocardial H/R injury from the perspectives of the inflammatory response (6), cell apoptosis (7) and cell signal transduction (8), but the exact molecular mechanism remains unknown. Therefore, it is important to explore the potential molecular mechanisms of myocardial IRI.

Emerging evidence has suggested that microRNAs (miRNAs/miRs) function as regulators in cells development, differentiation, immunity and cell cycle (9). In addition, miRNAs have been demonstrated to serve vital 
roles in improving the therapeutic outcomes of myocardial infarction (10), arrhythmia (11) and inhibition of atrial fibrillation (12). miR-155, as a typical multifunctional RNA, has been identified to be associated with homeostasis, atherogenesis, immune system and inflammation function (13). In addition, previous studies have observed that miR-155 was also involved in processes other than hematopoiesis and immune system, including cardiovascular disease (14), tumor and other pathological processes (15). It has previously been demonstrated that the inhibition of miR-155 ameliorated cardiac fibrosis in the process of angiotensin II-induced cardiac remodeling. In addition, previous data has identified that miR-155 functions as a vital moderator of cardiac damage and inflammation in atherosclerosis by repressing Bcl-6 in macrophages (16), and that miR-155 may aggravate ischemia-reperfusion injury via regulation of inflammatory cell recruitment and the respiratory oxidative burst (17). Downregulation of miR-155 may stimulate sevoflurane-mediated cardio protection against myocardial ischemia/reperfusion injury via binding to SIRT1 in mice (18). Furthermore, miR-155 may aggravate liver ischemia/reperfusion injury through limiting suppressor of cytokine signaling 1 in mice (19). However, data concerning how miR-155 functions in myocardial I/R injury, its potential molecular mechanism and the signaling pathway involved, are limited.

The present study identified that miR-155 was notably upregulated in a myocardial $\mathrm{H} / \mathrm{R}$ in vitro model. Following overexpression of miR-155, cell viability was markedly decreased, the number of apoptotic cells was significant increased, and the expression of apoptosis-associated proteins caspase 3 and caspase 9 were markedly upregulated; inhibition of miR-155 resulted in a reversal of all of these events. In addition, high expression levels of key proteins involved in the mitogen-activated protein kinase (MAPK)/JNK pathway caused by $\mathrm{H} / \mathrm{R}$ were attenuated by miR-155 inhibitor. BAG family molecular chaperone regulator 5 (BAG5), which was expressed at a decreased level in the H/R model, was confirmed to be a target of miR-155 and to be negatively regulated by miR-155. A co-transfection assay demonstrated that the overexpression of BAG5 may promote the mitigative effect of miR-155 inhibition on the cell damage induced by H/R. In addition, the high expression level of hypoxia-inducible factor 1- $\alpha$ (HIF-1 $\alpha$ ) and low expression level of von Hippel-Lindau protein (VHL) induced by H/R were suppressed by miR-155 inhibition. These data suggested that knockdown of miR-155 may alleviate the cell damage caused by $\mathrm{H} / \mathrm{R}$ by mediating BAG5 and the MAPK/JNK pathway. The function of miR-155/BAG5 on myocardial H/R injury represents a novel avenue of research for understanding the mechanism of $\mathrm{I} / \mathrm{R}$ and provides a theoretical reference for the identification of clinical therapeutic targets in the future.

\section{Materials and methods}

Construction of a myocardial ischemia model in vitro. Rat $\mathrm{H} 9 \mathrm{c} 2$ myocardial cells were obtained from the American Type Culture Collection and were used to construct the H/R model in vitro. H9c2 cells were washed with PBS and rendered quiescent in serum-free Dulbecco's modified Eagle's medium (DMEM, Sigma-Aldrich; Merck KGaA) for $24 \mathrm{~h}$ prior to experimentation. The cells were cultured in DMEM with $10 \%$ fetal bovine serum (FBS, Gibco; Thermo Fisher Scientific, Inc.) and gentamicin. Then, the medium was replaced with DMEM without FBS and the cells were cultured in a hypoxic incubator with $95 \% \mathrm{~N}_{2}$ and $5 \% \mathrm{CO}_{2}$ at $37^{\circ} \mathrm{C}$. Following culture in the hypoxic incubator for $6 \mathrm{~h}$, the cells were transferred to a normal incubator with $95 \% \mathrm{O}_{2}$ and $5 \% \mathrm{CO}_{2}$ at $37^{\circ} \mathrm{C}$ for $24 \mathrm{~h}$, in order to reoxygenate the cells.

Cell transfection. miR-155 mimic negative control (NC), miR-155 mimics, miR-155 inhibitor NC, and miR-155 inhibitors were synthesized by Shanghai GenePharma Co., Ltd. When cell coverage reached $80 \%$, Lipofectamine 3000 (Invitrogen; Thermo Fisher Scientific, Inc.) was used to transfect miR-155 mimic (200 nM, 5'-UUAAUGCUAAUCGUG AUAGGGGU-3'), inhibitor (100 nM, 3'-CCCUAUCACGAU UAGCAUUAAUU-3') and NC (100 nM, 5'-UUGUCCUAC ACCUCACAGUCCUG-3') into H9c2 cells following manufacturer's protocol to generate the knockout and overexpression cell models. Transfected cells were cultured at $37^{\circ} \mathrm{C}$ for $6 \mathrm{~h}$, followed by incubation with complete medium. After $24 \mathrm{~h}$, subsequent experiments were performed.

$R N A$ extraction and reverse transcription-quantitative polymerase chain reaction ( $R T-q P C R)$. Total cellular RNA was extracted from the treated cells using TRIzol ${ }^{\circledR}$ (Thermo Fisher Scientific, Inc.) according to manufacturer's protocol, and then reverse transcribed into cDNA using an iScript ${ }^{\mathrm{TM}}$ cDNA Synthesis kit (Bio-Rad Laboratories, Inc.) following the manufacturer's protocol. The mRNA/miRNA relative expression was assessed by qPCR using the SsoFast ${ }^{\mathrm{TM}}$ EvaGreen $^{\circledR}$ Supermix (Bio-Rad Laboratories, Inc.). The thermocycler conditions were as follows: $95^{\circ} \mathrm{C}$ for $2 \mathrm{~min}$, followed by 40 cycles at $95^{\circ} \mathrm{C}$ for $5 \mathrm{~min}, 95^{\circ} \mathrm{C}$ for $30 \mathrm{sec}$ and $60^{\circ} \mathrm{C}$ for $45 \mathrm{sec}$, and then $72^{\circ} \mathrm{C}$ for $30 \mathrm{~min}$. The $2^{-\Delta \Delta \mathrm{Cq}}$ method (20) was used to detect the relative fold-changes, and GAPDH and U6 were used as internal controls to detect mRNA and miRNA expression. The primer sequences were as follows: BAG5 forward, 5'-GCAAGTGGT TGGCTTCAGTG-3'; BAG5 reverse, 5'-CACGCATGATAA GTGCCTGC-3'; GAPDH forward, 5'-GCCAGCCTCGTC TCATAGAC-3'; GAPDH reverse, 5'-AGTGATGGCATGGAC TGTGG-3'; miR-155 forward 5'-AATGCTAATTGTGAT AGGGG-3'; miR-155 reverse, 5'-GAACATGTCTGCGTA TCTC-3'; U6 forward, 5'-CCTGCTTCGGCAGCACAT-3'; and U6 reverse, 5'-GCGTGAAGCGTTCCATG-3'.

Western blot analysis. Transfected cells were lysed using RIPA buffer (Invitrogen; Thermo Fisher Scientific, Inc.) with $1 \%$ protease inhibitor. A bicinchoninic acid kit (Beyotime Institute of Biotechnology) was used to measure the concentration of total proteins following the manufacturer's instruction. Equal amounts of proteins $(20 \mu \mathrm{g})$ were electrophoresed using 12\% SDS-PAGE gels, and then transferred onto PVDF membranes (Invitrogen; Thermo Fisher Scientific, Inc.). The membranes were blocked with $5 \%$ non-fat milk for $1 \mathrm{~h}$ at room temperature, and then incubated with primary antibodies purchased from Abcam: BAG5 (1:1,000; cat. no. ab97660), cleaved-caspase 3 (1:1,000; cat. no. ab2303), cleaved-caspase 9 (1:1,000; cat. no. ab2324), mitogen-activated protein kinase 11 (P38; 1:1,000; cat. no. ab170099), phosphorylated (p)-P38 
(1:1,000; cat. no. ab31828), JNK (1:1,000; cat. no. ab126424), p-JNK (1:1,000; cat. no. ab176662), HIF-1 $\alpha$ (1:1,000; cat. no. ab221610), VHL (1:4,000, ab140989) and GAPDH (1:1,000; cat. no. ab181602) overnight at $4^{\circ} \mathrm{C}$. The membranes were then washed with TBS $+0.1 \%$ Tween-20 3 times, and then cultured with the goat anti-rabbit IgG-HRP secondary antibody conjugated with horseradish peroxidase $(1: 10,000$, cat. no. ab6721, Abcam) for $1 \mathrm{~h}$ at room temperature. Finally, the protein bands were visualized using electrochemiluminescence according to manufacturer's protocol and analyzed using Quantity One software version 4.6.6 (Bio-Rad Laboratories, Inc.).

Cell viability assay. Cell proliferation was determined using a Cell Counting Kit-8 (CCK-8) assay. According to the manufacturer's protocol, cells were seeded onto 96 -well plates at the density of 1,000 cells/well. A total of $10 \mu \mathrm{l}$ CCK-8 solution was added to each well and incubated at $37^{\circ} \mathrm{C}$ for $1.5 \mathrm{~h}$; the cell activity was detected at $24 \mathrm{~h}$ intervals and the absorbance values at $450 \mathrm{~nm}$ wavelength were measured with a microplate spectrophotometer (Bio-Rad Laboratories, Inc.). All experiments were performed in triplicate.

Detection of apoptosis by Annexin V-fluorescein isothiocyanate (FITC)/propidium iodide (PI) double-staining. Cell apoptosis was examined using the Annexin-V-FITC Apoptosis Detection kit. The myocardial cells $\left(1-5 \times 10^{6}\right)$ were collected and washed in PBS following H/R treatment. Then, the myocardial cells were resuspended in binding buffer with $5 \mu \mathrm{l}$ Annexin V-FITC and $10 \mu \mathrm{l}$ PI double-stain, following the manufacturer's protocol. Finally, the samples were analyzed by a flow cytometer (BD Biosciences) and FlowJo version 7.6.3 software (Flow Jo LLC).

miR-155 target gene prediction and luciferase activity assay. Bioinformatics analysis tools including miRanda (21), miRWalk (22) and TargetScan (23) were used to predict the possible target mRNA of miR-155. A luciferase reporter gene assay was conducted using the Dual-Luciferase Reporter Assay System (Promega Corporation) according to the manufacturer's protocol. The 3'UTR segments of the BAG5 including the wild type (WT) or mutant (Mut) miR-155 binding sites were inserted into pGL3 luciferase vector (Promega Corporation). The transfected cells were seeded onto 24 -well plates at a density of $1 \times 10^{4} /$ well. 293 cells were then co-transfected with WT-BAG5/Mut-BAG5 plasmids and miR-155 mimic/NC by Lipofectamine ${ }^{\circledR} 3000$ (Invitrogen; Thermo Fisher Scientific, Inc.). Cultured for 48 h, transfected cells were harvested and the luciferase activity was detected using a Dual Luciferase ${ }^{\mathrm{TM}}$ reporting system (Promega Corporation). The luciferase activity was normalized to that of Renilla luciferase activity.

Statistical analysis. SPSS v.22.0 (IBM Corp.) software and GraphPad Prism v.5.0 (GraphPad Software, Inc.) were used to analyze the experimental data. Student's t-test was used to evaluate the difference between two groups. A one-way analysis of variance followed by Dunnett's post-hoc test, to compare all groups with the control group, or Tukey's post-hoc test, to compare all pairs of groups, was performed to assess the differences between multiple groups. $\mathrm{P}<0.05$ was considered to indicate a statistically significant difference.

\section{Results}

Increased expression of miR-155 is exhibited in myocardial cells exposed to $H / R$. To explore the function of miR-155 in myocardial IRI, a model of $\mathrm{H} / \mathrm{R}$ in myocardial cells was first established to simulate the cells in myocardial IRI in vivo. The RT-qPCR data indicated that miR-155 was notably upregulated in the $\mathrm{H} / \mathrm{R}$ model cells compared with the untreated cells (Fig. 1A; $\mathrm{P}<0.01$ ). Then, miR-155 mimics and inhibitors were applied to regulate the expression of miR-155. As indicated in Fig. 1B, the expression of miR-155 was successfully up- or downregulated by miR-155 mimics or inhibitors, respectively. Similarly, in the H/R model, the results demonstrated that the relative expression of miR-155 was up- or downregulated following transfection with the miR-155 mimics or inhibitors, respectively (Fig. $1 \mathrm{C} ; \mathrm{P}<0.01$ ). These results suggested that miR-155 was highly expressed in myocardial cells in the H/R model and was successfully regulated by the miR-155 mimics and inhibitors.

Effect of miR-155 on the activity and apoptosis of injured $H / R$-stimulated myocardial cells. To additionally identify the type of cell injury induced by H/R stimulation, a CCK-8 assay was performed to examine cell viability. The results indicated that cell viability was significantly decreased in the H/R model compared with the sham group. Following transfection with the miR-155 mimic, the cell viability was additionally decreased compared with the non-transfected $\mathrm{H} / \mathrm{R}$ cells. However, following transfection with the miR-155 inhibitor, cell viability was markedly increased compared with the non-transfected H/R cells (Fig. $2 \mathrm{~A} ; \mathrm{P}<0.05$ ).

Flow cytometry analysis was conducted to detect the levels of cell apoptosis. As demonstrated in Fig. 2B and C, the number of apoptotic cells was increased $>2$-fold in the $H / R$ cells compared with the sham group. Following transfection with the miR-155 mimic, the number of apoptotic cells was markedly increased compared with the non-transfected H/R model cells. However, transfection with the miR-155 inhibitor resulted in a marked decrease in the number of apoptotic cells compared with the non-transfected H/R model cells.

Western blot analysis was used to identify the expression levels of the apoptotic proteins cleaved caspase 3 and 9 following different treatments. The data from Fig. 2D and E indicated that the expression of cleaved caspase- 3 and 9 were markedly increased in the H/R-treated cells compared with the sham group. Following transfection with the miR-155 mimic, the expression levels of cleaved caspase-3 and 9 were markedly increased compared with the non-transfected H/R group. However, following transfection with miR-155 mimic, the expression of cleaved caspase- 3 and 9 were markedly decreased compared with the non-transfected H/R group. Based on these results, it was identified that the overexpression of miR-155 aggravated myocardial cell injury in a H/R model. Conversely, miR-155 downregulation reversed these effects.

miR-155 directly targets BAG5 to function in $H / R$. It is well-known that miRNAs serve various roles by regulating 

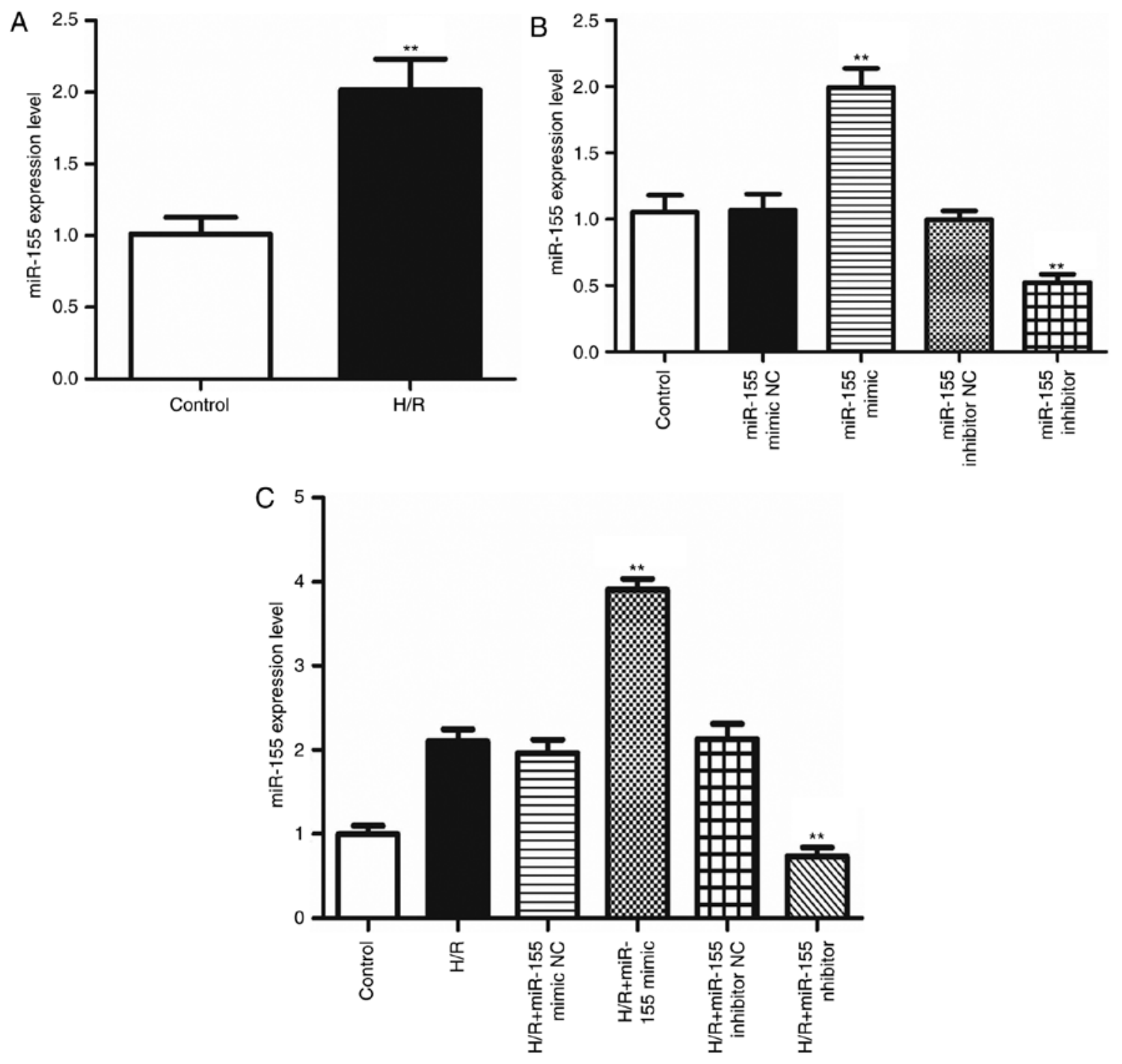

Figure 1. Expression of miR-155 is increased in a myocardial H/R model. (A) The miRNA expression of miR-155 in the H/R model was significantly upregulated compared with the control group, ${ }^{* *} \mathrm{P}<0.01$. (B) miR-155 mimics and inhibitors markedly increased and decreased the expression of miR-155, respectively, compared with the NC group. ${ }^{* *} \mathrm{P}<0.01$. (C) The miRNA expression of miR-155 in the H/R model was markedly up- or downregulated following transfection with miR-155 mimics or inhibitors, compared with the NC group. ${ }^{* *} \mathrm{P}<0.01$. miR, microRNA; H/R, hypoxia/reoxygenation; NC, negative control.

their downstream target genes. Bioinformatics analysis tools including miRanda, miRWalk and TargetScan were applied to predict the possible target mRNA of miR-155. BAG5 was predicted as a target of miR-155. Each miRNA was predicted to have multiple target mRNAs. BAG5 was selected due to its involvement in a number of important physiological and pathological processes, including the development of tumors and the treatment of Parkinson's disease (24), but few studies have been performed on ischemia-reperfusion injury. Therefore, the present study focused on BAG5. The sequences of WT-BAG5, Mut-BAG5 and miR-155 are presented in Fig. 3A. The data from luciferase reporter assay (Fig. 3D) indicated that the luciferase activity was decreased in WT-BAG5 group following transfection with the miR-155 mimic, but that the luciferase activity was nearly unchanged in the Mut-BAG5 group, which confirmed the association between BAG5 and miR-155. Then, the protein expression level of BAG5 in the H/R-treated cells was examined. The data demonstrated that BAG5 was downregulated in the H/R group compared with the sham group (Fig. 3B and $\mathrm{C} ; \mathrm{P}<0.01$ ). Finally, the mRNA and protein expression levels of BAG5 were detected in the miR-155 NC and mimic groups. In the miR-155 mimic group, it was identified that the expression of BAG5 was significant decreased at both mRNA and protein levels compared with the NC group (Fig. 3E-G; $\mathrm{P}<0.01$ ). These results indicated that BAG5, which was expressed at a decreased level in the H/R-treated group, was a confirmed target of miR-155 and was negatively regulated by miR-155.

Cell injury caused by H/R is attenuated by miR-155 inhibition partly through MAPK pathway. The MAPK signaling pathway is one of the most important signal transduction systems in vivo, and is involved in mediating various physiological and pathological processes such as cell growth, development, division and differentiation. MAPK subfamilies in mammals primarily include ERK, JNK and P38. However, JNK and P38 have been demonstrated to regulate cell apoptosis (25), proliferation (26) or pressor response (27). In addition, JNK and P38 have been revealed to participate the process of ischemia/reperfusion injury in rats (28). Therefore, p-JNK and p-P38 were selected 

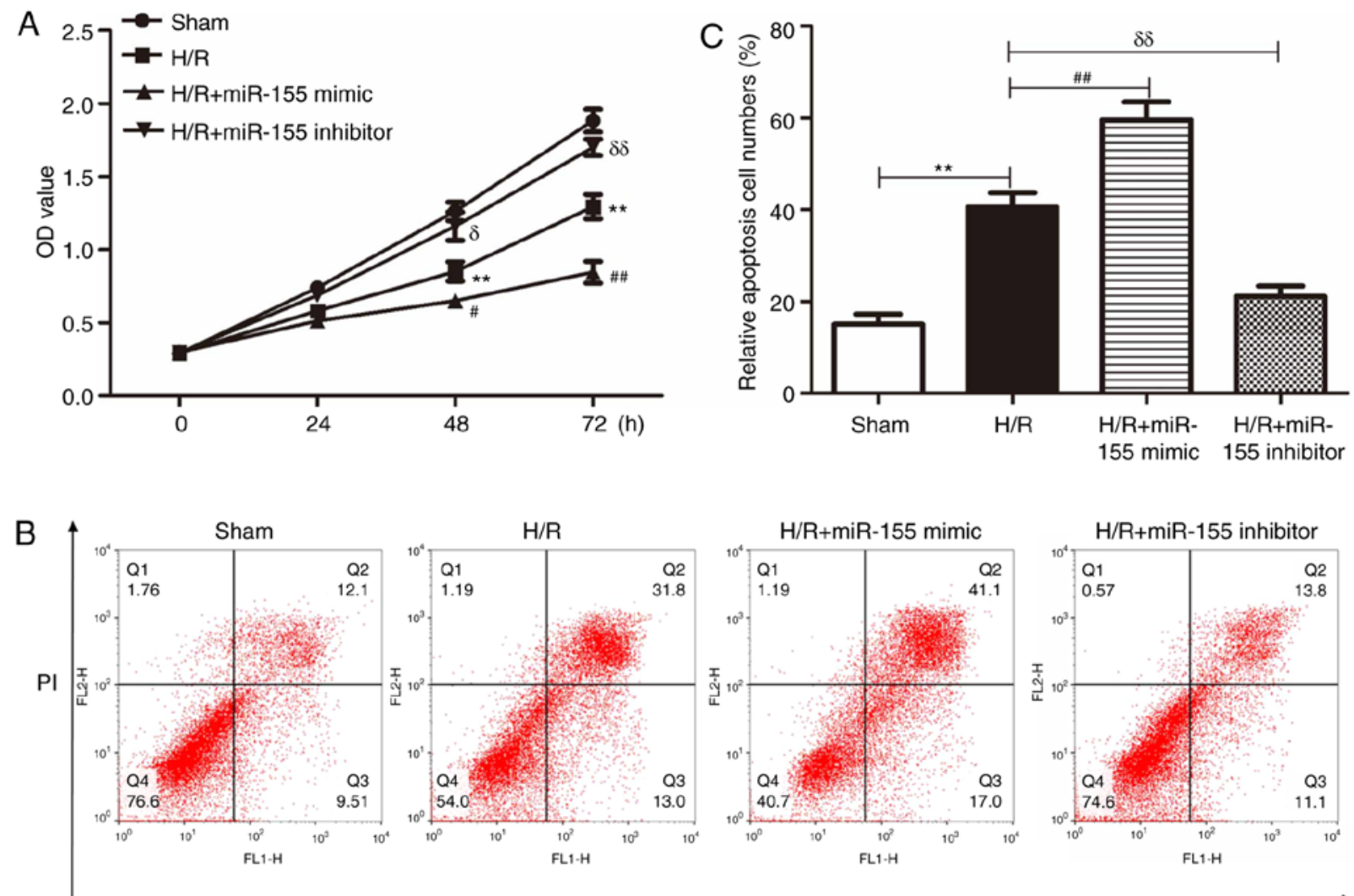

Annexin V FITC
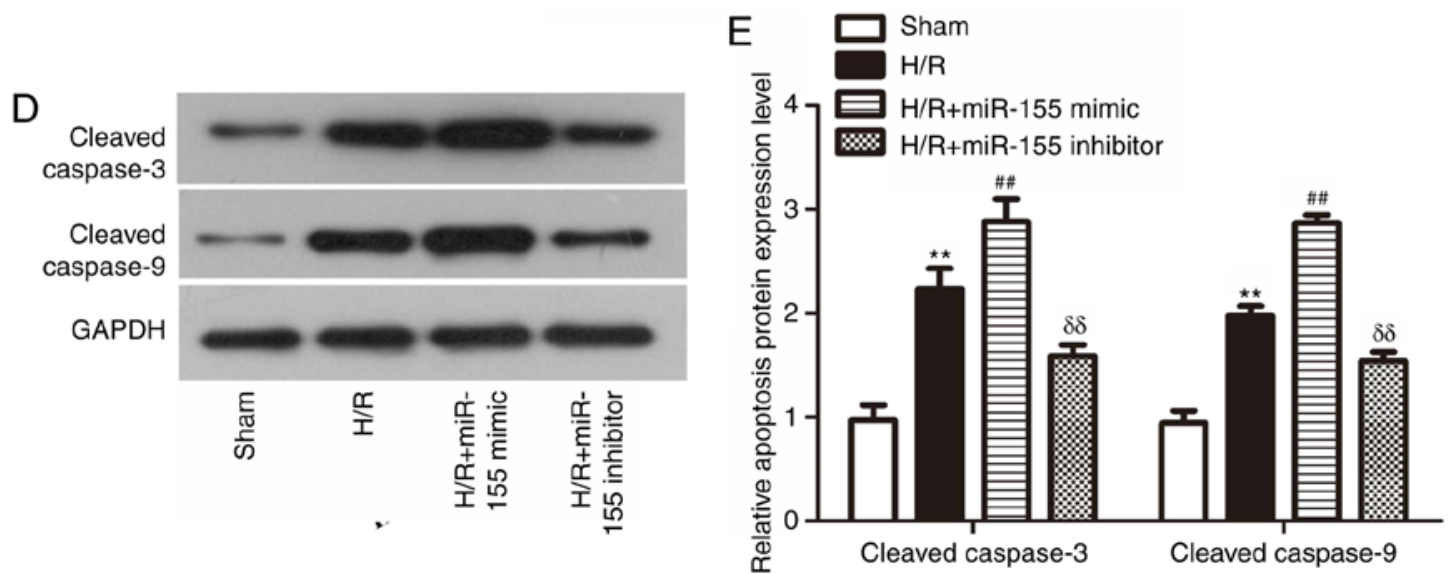

Figure 2. Effects of miR-155 on the H/R model. (A) The effect of miR-155 on the proliferation of myocardial cells in the H/R model was measured by CCK-8 assay following transfection with miR-155 mimics or inhibitors. ${ }^{* *} \mathrm{P}<0.01$ vs. sham. ${ }^{\#} \mathrm{P}<0.05$ and ${ }^{\# \#} \mathrm{P}<0.01 \mathrm{H} / \mathrm{R}$ vs. $\mathrm{H} / \mathrm{R}+{ }^{\mathrm{miR}} \mathrm{R}-155 \mathrm{mimic} .{ }^{\delta} \mathrm{P}<0.05$ and ${ }^{\delta \delta} \mathrm{P}<0.01$ H/R vs. H/R + miR-155 inhibitor. (B) Flow cytometry analysis indicated the number of apoptotic cells following transfection with miR-155 mimics or inhibitors in the H/R model. (C) Quantified cell apoptosis data. ${ }^{* *} \mathrm{P}<0.01$ sham vs. H/R. ${ }^{\# \#} \mathrm{P}<0.01 \mathrm{H} / \mathrm{R}$ vs. H/R + miR-155 mimic. ${ }^{\delta \delta} \mathrm{P}<0.01 \mathrm{H} / \mathrm{R}$ vs. $\mathrm{H} / \mathrm{R}+\mathrm{miR}-155$ inhibitor. (D) The expression levels of pro-apoptotic proteins cleaved-caspase 3 and cleaved-caspase 9 were measured by western blot analysis following transfection with miR-155 mimics or inhibitors in the H/R model. $\mathrm{P}<0.01$. (E) Quantified densitometric data from the western blot analysis. ${ }^{* *} \mathrm{P}<0.01,{ }^{\# \prime} \mathrm{P}<0.01$ $\mathrm{H} / \mathrm{R}$ vs. $\mathrm{H} / \mathrm{R}+$ miR-155 mimic. ${ }^{\delta} \mathrm{P}<0.01 \mathrm{H} / \mathrm{R}$ vs. $\mathrm{H} / \mathrm{R}+$ miR-155 inhibitor. miR, microRNA; H/R, hypoxia/reoxygenation; FITC, fluorescein isothiocyanate; $\mathrm{PI}$, propidium iodide.

for examination in the present study. In the H/R model, it was identified that the protein expression levels of p-P38 and p-JNK were significantly increased. Following transfection with the miR-155 inhibitor, the protein expression of p-P38 and p-JNK were markedly decreased (Fig. 4A and B; $\mathrm{P}<0.01$ ). In addition, the increased ratios of phosphorylated to total P38 and JNK proteins in the H/R model was suppressed following miR-155 inhibition. This result suggested that the overexpression of p-P38 and p-JNK in the H/R model cells was inhibited following transfection with the miR-155 inhibitor, suggesting that miR-155 functions as a regulator in myocardial $\mathrm{H} / \mathrm{R}$ injury, partly through the MAPK signaling pathway.

Overexpression of BAG5 enhances the effect of $m i R-155$ inhibitor on H/R-induced myocardial cells impairment. Following on from the aforementioned results, it was demonstrated that miR-155 silencing promoted the protective effects on myocardial injury caused by H/R. However, the effects of 


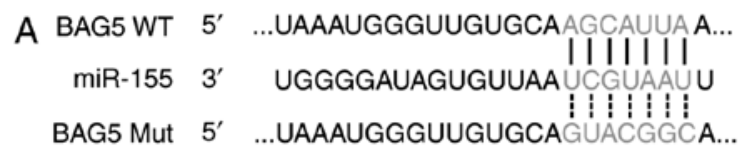

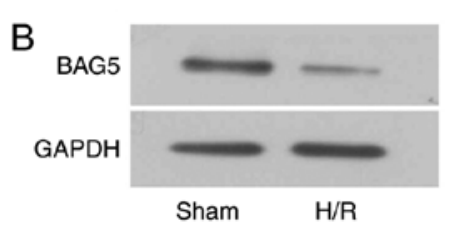

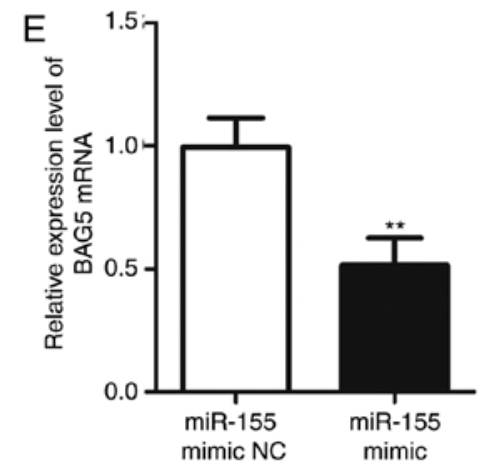

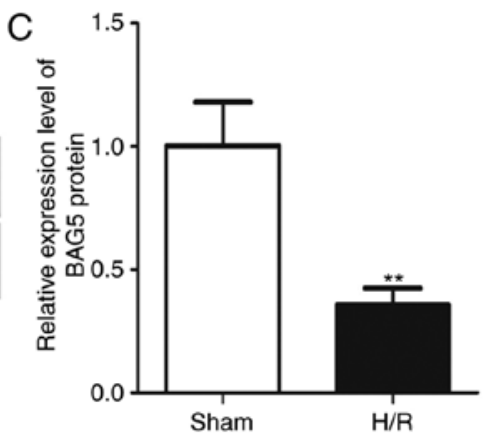
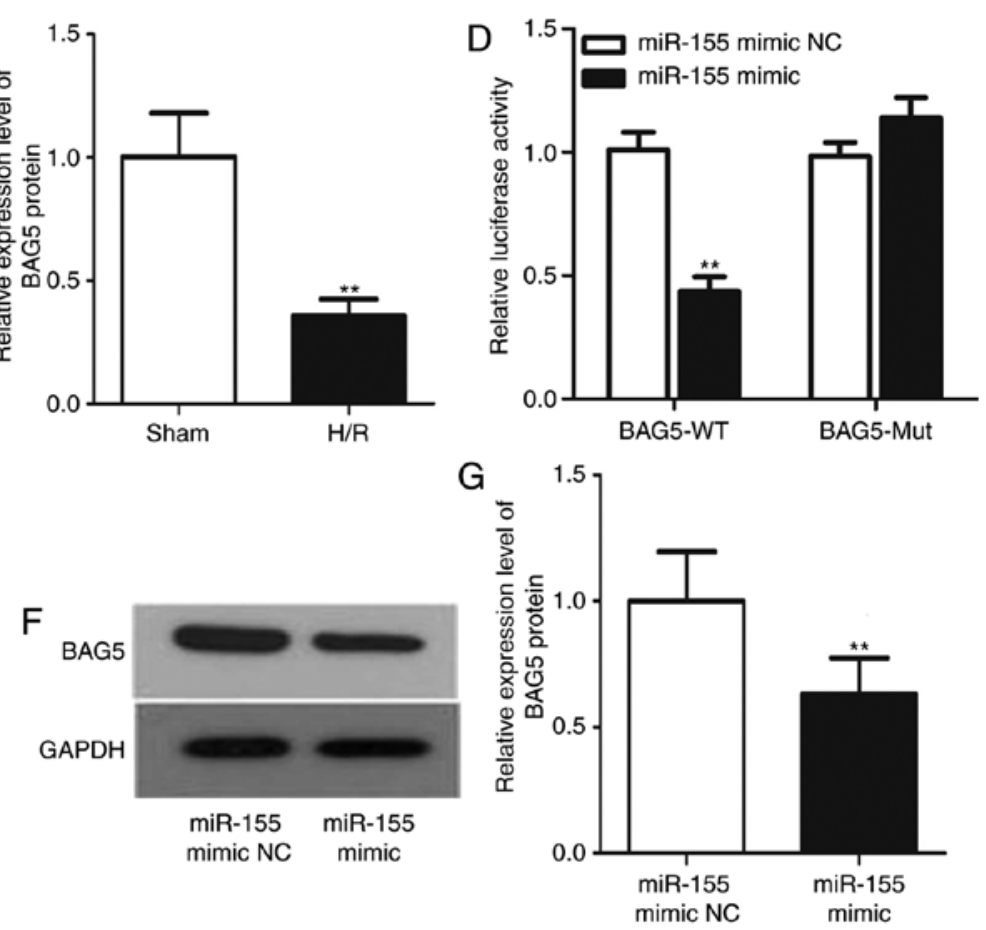

Figure 3. BAG5 is negatively regulated by miR-155 and is expressed at lower levels in the H/R model. (A) The sequences of 3'-UTR-WT BAG5, 3'-UTR-Mut BAG5 and miR-155. (B) The expression level of BAG5 was detected by western blot analysis. (C) Quantifieddensitometric western blot analysis data. ${ }^{* *} \mathrm{P}<0.01$. (D) The relative luciferase activity in Mut BAG5 and WT BAG5 were determined by luciferase reporter assay following transfection with miR-155 mimic or NC. ${ }^{* *} \mathrm{P}<0.01$. The (E) mRNA and $(\mathrm{F})$ protein expression of BAG5 in the $\mathrm{H} / \mathrm{R}$ model following transfection with miR-155 mimic or NC. ${ }^{* *} \mathrm{P}<0.01$. (G) Quantified densitometric western blot analysis data. BAG5, BAG family molecular chaperone regulator 5; miR, microRNA; H/R, hypoxia/reoxygenation; UTR, untranslated region; WT, wild type; Mut, mutant; NC, negative control.
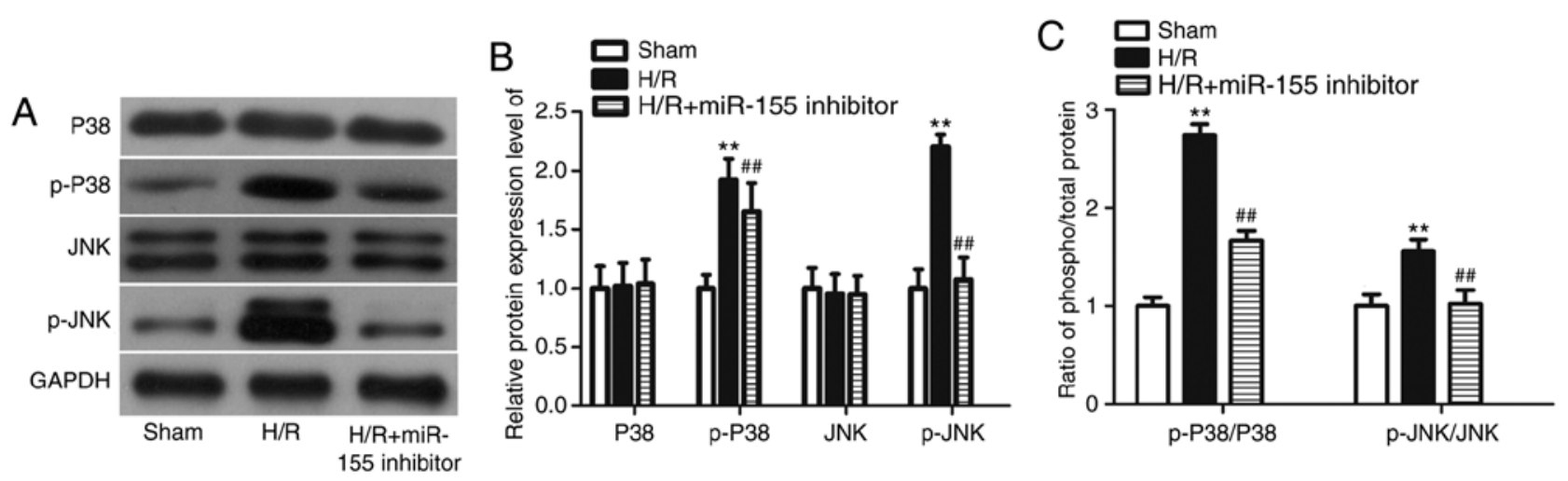

Figure 4. Expression of MAPK pathway-associated proteins was determined by western blot analysis. (A) The expression of p-P38 and p-JNK were significantly upregulated in H/R model and were downregulated following transfection with miR-155 inhibitor in the H/R model. (B) Quantified densitometric western blot analysis data. (C) The ratio of phosphorylated to total P38 and JNK proteins in the H/R model following treatment with the miR-155 inhibitor. ${ }^{* *} \mathrm{P}<0.01$ vs. sham, ${ }^{\# \#} \mathrm{P}<0.01$ vs. H/R. miR, microRNA; H/R, hypoxia/reoxygenation; p-, phosphorylated. $\mathrm{P} 38$, mitogen-activated protein kinase 11.

miR-155/BAG5 on myocardial cell injury caused by H/R were unclear. The miR-155 inhibitor and pcDNA3.1-BAG5 were co-transfected into the H9c2 cells to evaluate the effect of miR-155/BAG5 on cells viability and apoptosis. As presented in Fig. 5A, the expression of BAG5 was significantly upregulated after transfection with pcDNA3.1-BAG5 compared with the sham or empty vector. The CCK- 8 assay data indicated that
BAG5 may promote the mitigative effect of miR-155 inhibition on the H9c2 cells damage induced by $\mathrm{H} / \mathrm{R}$ (Fig. 5B; $\mathrm{P}<0.05$ ). The flow cytometry data demonstrated that overexpression of BAG5 promoted the inhibitory effect of miR-155 inhibitor on the apoptosis of $\mathrm{H} 9 \mathrm{c} 2$ cells (Fig. $5 \mathrm{C}$ and $\mathrm{D} ; \mathrm{P}<0.01$ ).

It is well-known that HIF regulates cellular protection against decreased oxygen delivery via modulating cellular pathways 

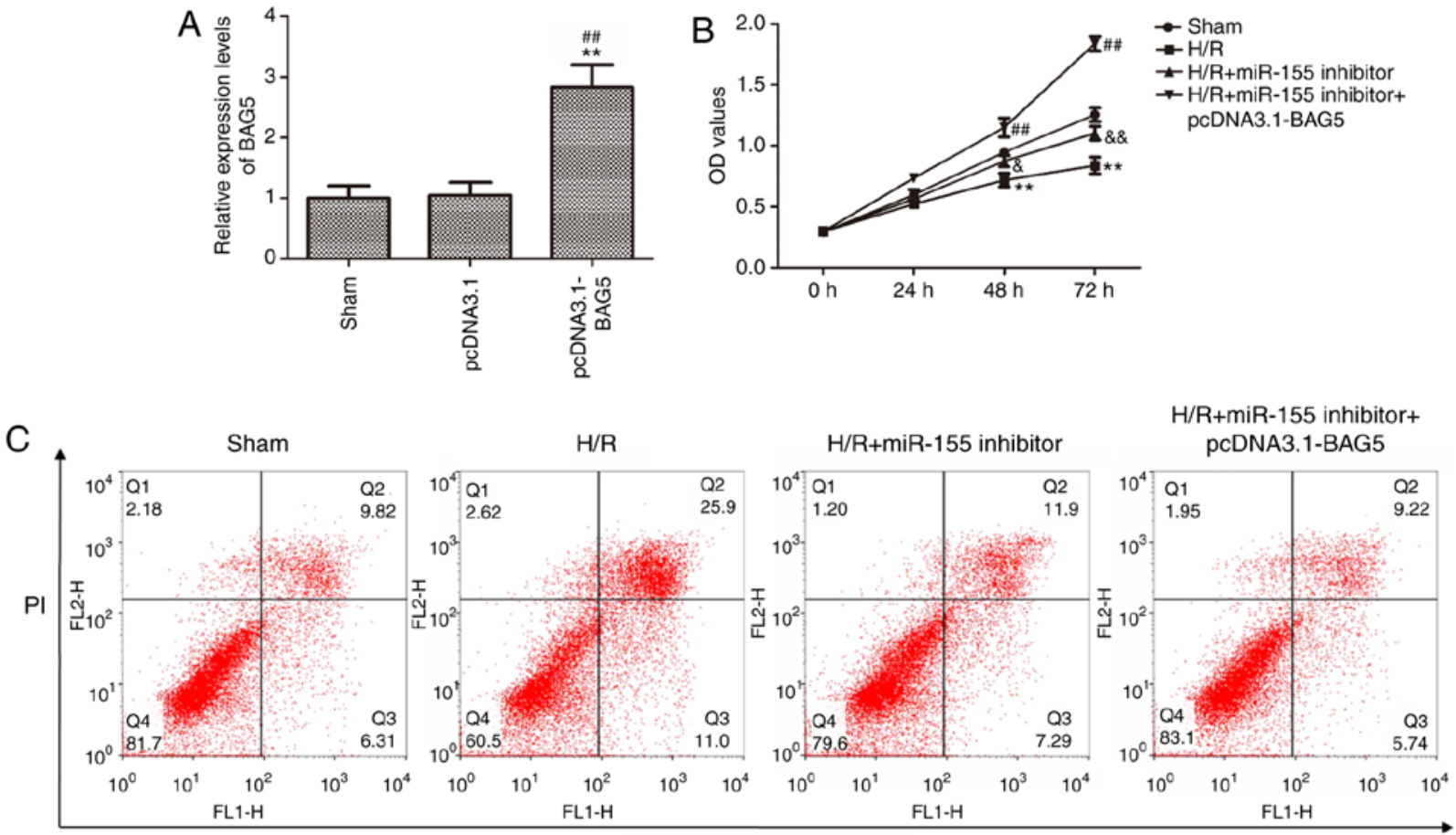

Annexin V FITC

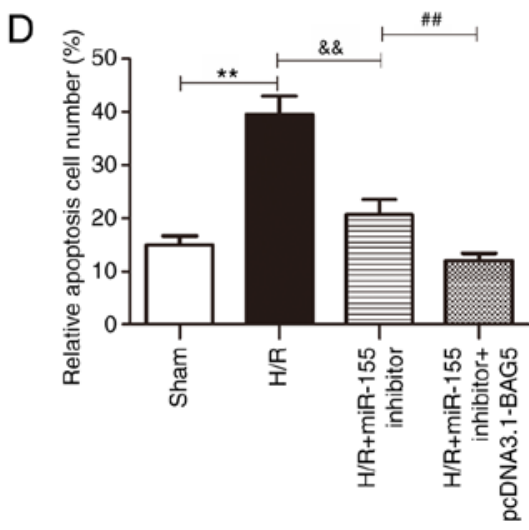

Figure 5. Overexpression of BAG5 enhances the protective effect of the miR-155 inhibitor on myocardial cells damage in the H/R model. (A) The transfection efficiency of pcDNA3.1-BAG5 was measured by reverse transcription-quantitative polymerase chain reaction. ${ }^{* *} \mathrm{P}<0.01 \mathrm{vs}$. sham, ${ }^{\# \#} \mathrm{P}<0.01 \mathrm{vs}$. pcDNA3.1. (B) Cell viability was detected using CCK-8 assay following transfection with miR-155 inhibitor and pcDNA3.1-BAG5 in the H/R model. ${ }^{\circ} \mathrm{P}<0.05$ and ${ }^{\delta \delta} \mathrm{P}<0.01$ vs. $\mathrm{H} / \mathrm{R},{ }^{* *} \mathrm{P}<0.01$ vs. sham, ${ }^{\# \#} \mathrm{P}<0.01$ vs. $\mathrm{H} / \mathrm{R}+\mathrm{miR}-155$ inhibitor. (C) The level of cell apoptosis was measured by flow cytometry following transfection with miR-155 inhibitor and pcDNA3.1-BAG5 in the H/R model. (D) Quantified flow cytometry data. ${ }^{* *} \mathrm{P}<0.01$ vs. sham, ${ }^{\# \#} \mathrm{P}<0.01$ vs. H/R + miR-155 inhibitor, ${ }^{\delta \delta} \mathrm{P}<0.01$ vs. H/R. BAG5, BAG family molecular chaperone regulator 5; miR, microRNA; H/R, hypoxia/reoxygenation; FITC, fluorescein isothiocyanate; PI, propidium iodide.

and functions $(29,30)$. The oxygen-regulated HIF-1 $\alpha$ subunit is continuously synthesized in cells but promptly degraded in the presence of oxygen following hydroxylation. Hydroxylated HIF is targeted for proteasomal degradation after binding to the VHL E3 ubiquitin ligase (31). Therefore, the expression levels of HIF-1 $\alpha$ and VHL in the H/R model of the present study were detected following different treatment. In the H/R model, the expression of HIF-1 $\alpha$ was increased, while the expression of VHL was decreased compared with the sham group. However, this phenomenon was reversed after transfection with miR-155 inhibitor or miR-155 inhibitor + pcDNA3.1-BAG5 (Fig. 6A and $\mathrm{B} ; \mathrm{P}<0.01$ ). Altogether, the results demonstrated that co-transfection with pcDNA3.1-BAG5 and miR-155 inhibitor decreased the level of myocardial cells apoptosis and increased the level of proliferation compared with the cells only transfected with miR-155 inhibitor, through altering the expression patterns of HIF-1 $\alpha$ and VHL, suggesting that depletion of miR-155 may attenuate cell damage in the myocardial $\mathrm{H} / \mathrm{R}$ model by targeting BAG5 and regulating the expression of HIF-1 $\alpha$ and VHL.

\section{Discussion}

Reperfusion injury is one of the most significant complications in the recovery of ischemic myocardial blood flow. The toxic and side effects of reperfusion injury are caused by numerous complex pathological mechanism(32). Variousforms of myocardial IRI have been identified, including reperfusion induced arrhythmias, myocardial coma, microvascular obstruction and fatal myocardial reperfusion injury; only the first two of these are reversible (33). The underlying mechanisms of IRI are not 

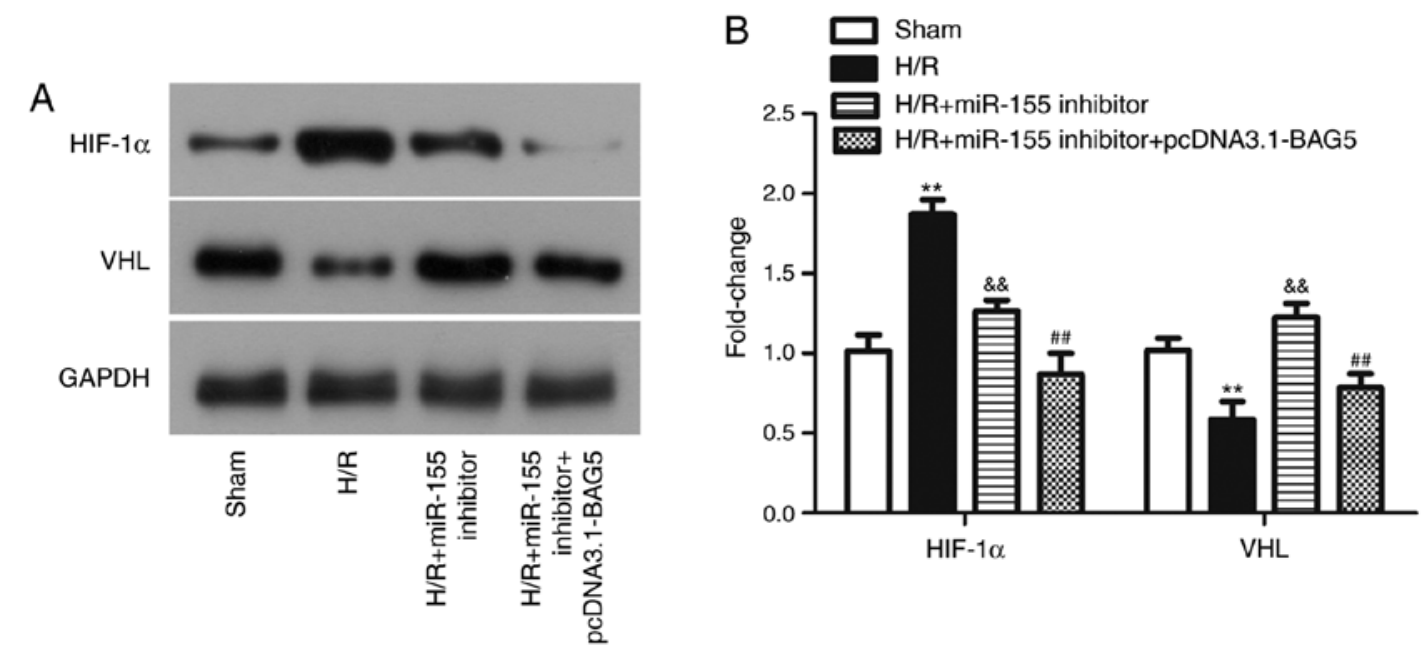

Figure 6. Expression of HIF-1 $\alpha$ and VHL were assessed by western blot analysis in the H/R model following different treatments. (A) The expression of HIF-1 $\alpha$ and VHL were measured by western blot analysis. (B) Quantified densitometric western blot analysis data. ${ }^{* *} \mathrm{P}<0.01$ sham vs. H/R. ${ }^{\# \#} \mathrm{P}<0.01 \mathrm{H} / \mathrm{R}+$ miR-155 inhibitor vs. H/R + miR-155 inhibitor + pcDNA3.1-BAG5. ${ }^{\delta \delta} \mathrm{P}<0.01 \mathrm{H} / \mathrm{R}$ vs. $\mathrm{H} / \mathrm{R}+$ miR-155 inhibitor. HIF-1 $\alpha$, hypoxia-inducible factor $1-\alpha$; VHL, von Hippel-Lindau.

entirely clear (34), but the modification of molecular, cellular and tissues through processes such as oxidative stress, cell death, neurohumoral activation and inflammation are regarded critical in the development of IRI $(35,36)$. Although a complete understanding of myocardial IRI completely remains a challenge, active reperfusion remains the most important method and is being vigorously initiated. The present study identified that depletion of miR-155 improved myocardial cells injury caused by H/R. The data from the CCK-8 assay demonstrated that the downregulation of miR-155 enhanced the myocardial cells activity, the flow cytometry analysis data revealed that miR-155 inhibition attenuated myocardial cells apoptosis, and the western blot analysis data indicated that depletion of miR-155 decreased the protein expression levels of key apoptotic proteins caspase 3 and caspase 9. Using Bioinformatics prediction software programs and a dual-luciferase reporter assay, BAG5 was confirmed as a direct functional target of miR-155. In addition, BAG5 was downregulated in the H/R model cells and was negatively regulated by miR-155. The data from the co-transfection assay suggested that overexpression of BAG5 could promote the protective effect of miR-155 inhibitor on cell damage caused by $\mathrm{H} / \mathrm{R}$ through regulating the expression of HIF-1 $\alpha$ and VHL. Taken together, the results of the present study indicated that the inhibition of miR-155 expression improved the outcomes of myocardial IRI through mediating BAG5 and the MAPK signaling pathway.

miR-155, as a multifunctional RNA, has been identified to function in numerous pathological and physiological processes including viral infection (37), hematopoietic lineage differentiation (38), cardiovascular disease (39), immunity (40), cancer (41), inflammation (42) and Down syndrome (43). In the present study, it was identified that miR-155 was highly expressed in the myocardial H/R model, and that overexpression of miR-155 decreased myocardial cells activity, increased the number of apoptotic cells and increased the expression of pro-apoptotic proteins. However, silencing miR-155 improved myocardial cell viability, decreased the number of apoptotic cells and attenuated the expression levels of pro-apoptotic proteins. These results indicated that miR-155 functioned as a crucial modulator in myocardial IRI.

It is well-known that mature miRNA regulate gene expression through binding to complementary sites in the 3'-untranslated region of their target genes. According to the analysis results of bioinformatics prediction software, BAG5 was selected as a specific target gene of miR-155. BAG5, as an important member of the BAG family, has been reported to demonstrate anti-apoptotic effects in prostate cancer (44). A previous study identified that BAG5 protected neuronal cells from amyloid $\beta$-induced cell death in Alzheimer's disease (45), and it has been demonstrated that BAG5 decreased the degradation of PTEN and maintained its stability through an ubiquitylation-dependent pathway (46). It has previously been observed that BAG5 may promote the accumulation of mutant p53 in tumors, and increase the gain-of-functions of mutant p53 (47). The study conducted by Kalia et al (48) established that BAG5 may inhibit parkin and enhance dopaminergic neuron degeneration. In addition, it has been suggested that BAG5 may function together with miR-127-3p to inhibit epithelial ovarian cancer cell growth (49). The present study demonstrated that BAG5 was a direct target gene of miR-155, and was downregulated in H/R. Concomitantly, overexpression of miR-155 was demonstrated to attenuate the levels of BAG5 expression, and overexpression of BAG5 in the H/R model promoted the protective effects of the miR-155 inhibitor on H/R-induced cell damage. A limitation of the present study was that only the basic phenomena were identified, and that cell models in vitro cannot fully simulate in vivo scenarios; further in vivo experiments are required to explore the specific mechanism.

In the process of myocardial $\mathrm{H} / \mathrm{R}$, the pathophysiological significance of myocardial cell apoptosis is important. It has been suggested that the JNK/MAPK signaling pathway is associated with various pathophysiological processes during apoptosis and oxidative stress. Bourke et al (50) demonstrated that, in an in vitro H/R injury model, anti-phospholipid antibodies enhanced the levels of apoptosis of newborn rat cardiac myocytes through p38 MAPK. It has previously been observed 
that myocardial cells were protected from H/R injury through inhibiting JNK (51). In concordance with previous data, the present study identified that the expression levels of MAPK/JNK-associated proteins p-P38 and p-JNK were markedly decreased following transfection with the miR-155 inhibitor, indicating that miR-155 regulated myocardial H/R injury through the MAPK/JNK signaling pathway.

The results from the present study demonstrated that miR-155/BAG5 function in myocardial H/R injury partly through the MAPK/JNK signaling pathway, suggesting that miR-155/BAG5 may be promising targets for the development of novel therapies for myocardial IRI.

In conclusion; the present study demonstrated that miR-155 was highly expressed in a myocardial H/R model, leading to decreased levels of myocardial cell proliferation and increased levels of apoptosis. Bioinformatics prediction software and a luciferase reporter assay confirmed that BAG5 was a specific target gene of miR-155. In addition, co-transfection of the miR-155 inhibitor and pcDNA3.1-BAG5 further indicated that miR-155 inhibition alleviated the myocardial H/R injury through targeting BAG5, via the MAPK/JNK signaling pathway.

\section{Acknowledgements}

Not applicable.

\section{Funding}

No funding was received.

\section{Availability of data and materials}

The datasets used and /or analyzed during the current study are available from the corresponding author on reasonable request.

\section{Authors' contributions}

JX conceived, designed, and performed the experiments and edited the manuscript. QL performed the experiments and provided technical support. BL analyzed the data and edited the manuscript. NL conceived, supervised and supported the study and edited the manuscript. All authors have read, revised and approved the final version of the manuscript. All authors agree to be accountable for all aspects of the work in ensuring that questions related to the accuracy or integrity of the work are appropriately investigated and resolved.

\section{Ethics approval and consent to participate}

Not applicable.

\section{Patient consent for publication}

Not applicable.

\section{Competing interests}

The authors declare that they have no competing interests.

\section{References}

1. Jennings RB, Sommers HM, Smyth GA, Flack HA, Linn H: Myocardial necrosis induced by temporary occlusion of a coronary artery in the dog. Arch Pathol 70: 68-78, 1960.

2. Eltzschig HK and Eckle T: Ischemia and reperfusion-from mechanism to translation. Nat Med 17: 1391-401, 2011.

3. Sun G, Lu Y, Li Y, Mao J, Zhang J, Jin Y, Li Y, Sun Y, Liu L and Li L: miR-19a protects cardiomyocytes from hypoxia/reoxygenation-induced apoptosis via PTEN/PI3K/p-Akt pathway. Biosci Rep 37: BSR20170899, 2017.

4. Wang X, Ha T, Hu Y, Lu C, Liu L, Zhang X, Kao R, Kalbfleisch J, Williams D and Li C: MicroRNA-214 protects against hypoxia/reoxygenation induced cell damage and myocardial ischemia/reperfusion injury via suppression of PTEN and Bim1 expression. Oncotarget 7: 86926-86936, 2016.

5. Qiu R, Li W and Liu Y: MicroRNA-204 protects H9C2 cells against hypoxia/reoxygenation-induced injury through regulating SIRT1-mediated autophagy. Biomed Pharmacother 100: 15-19, 2018.

6. Kawaguchi M, Takahashi M, Hata T, Kashima Y, Usui F, Morimoto H, Izawa A, Takahashi Y, Masumoto J, Koyama J, et al: Inflammasome activation of cardiac fibroblasts is essential for myocardial ischemia/reperfusion injury. Circulation 123: 594-604, 2011.

7. Feng R, Liu J, Wang Z, Zhang J, Cates C, Rousselle T, Meng Q and $\mathrm{Li} \mathrm{J}$ : The structure-activity relationship of ginsenosides on hypoxia-reoxygenation induced apoptosis of cardiomyocytes. Biochem Biophys Res Commun 494: 556-568, 2017.

8. Li Y, Shi X, Li J, Zhang M and Yu B: Knockdown of KLF11 attenuates hypoxia/reoxygenation injury via JAK2/STAT3 signaling in H9c2. Apoptosis 22: 510-518, 2017.

9. Tutar Y: miRNA and cancer; computational and experimental approaches. Curr Pharm Biotechnol 15: 429, 2014

10. Lesizza P, Prosdocimo G, Martinelli V, Sinagra G, Zacchigna S and Giacca M: Single-dose intracardiac injection of Pro-regenerative MicroRNAs improves cardiac function after myocardial infarction. Circ Res 120: 1298-1304, 2017.

11. Li YG, Zhang PP, Jiao KL and Zou YZ: Knockdown of microRNA-181 by lentivirus mediated siRNA expression vector decreases the arrhythmogenic effect of skeletal myoblast transplantation in rat with myocardial infarction. Microvasc Res 78: 393-404, 2009.

12. Wang J, Bai Y, Li N, Ye W, Zhang M, Greene SB, Tao Y, Chen Y, Wehrens XH and Martin JF: Pitx2-microRNA pathway that delimits sinoatrial node development and inhibits predisposition to atrial fibrillation. Proc Natl Acad Sci USA 111: 9181-6, 2014.

13. Rodriguez A, Vigorito E, Clare S, Warren MV, Couttet $P$, Soond DR, van Dongen S, Grocock RJ, Das PP, Miska EA, et al: Requirement of bic/microRNA-155 for normal immune function. Science 316: 608-611, 2007.

14. Elton TS, Selemon H, Elton SM and Parinandi NL: Regulation of the MIR155 host gene in physiological and pathological processes. Gene 532: 1-12, 2013

15. Faraoni I, Antonetti FR, Cardone J and Bonmassar E: miR-155 gene: A typical multifunctional microRNA. Biochim Biophys Acta 1792: 497-505, 2009.

16. Nazari-Jahantigh M, Wei Y, Noels H, Akhtar S, Zhou Z, Koenen RR, Heyll K, Gremse F, Kiessling F, Grommes J, et al: MicroRNA-155 promotes atherosclerosis by repressing Bcl6 in macrophages. J Clin Invest 122: 4190-202, 2012.

17. Eisenhardt SU, Weiss JB, Smolka C, Maxeiner J, Pankratz F, Bemtgen X, Kustermann M, Thiele JR, Schmidt Y, Bjoern Stark G, et al: MicroRNA-155 aggravates ischemia-reperfusion injury by modulation of inflammatory cell recruitment and the respiratory oxidative burst. Basic Res Cardiol 110: 32, 2015.

18. Nederlof R, Eerbeek O, Hollmann MW, Southworth R and Zuurbier CJ: Targeting hexokinase II to mitochondria to modulate energy metabolism and reduce ischaemia-reperfusion injury in heart. Br J Pharmacol 171: 2067-2079, 2014.

19. Tan L, Jiang W, Lu A, Cai H and Kong L: miR-155 aggravates liver Ischemia/reperfusion injury by suppressing SOCS1 in mice. Transplant Proc 50: 3831-3839, 2018.

20. Livak KJ and Schmittgen TD: Analysis of relative gene expression data using real-time quantitative PCR and the 2(-Delta Delta C(T)) method. Methods 25: 402-408, 2001.

21. Enright AJ, John B, Gaul U, Tuschl T, Sander C and Marks DS: MicroRNA targets in drosophila. Genome Biol 5: R1, 2003. 
22. Dweep H and Gretz N: miRWalk2.0: A comprehensive atlas of microRNA-target interactions. Nat Methods 12: 697, 2015.

23. Lewis BP, Burge CB and Bartel DP: Conserved seed pairing, often flanked by adenosines, indicates that thousands of human genes are microRNA targets. Cell 120: 15-20, 2005.

24. Kalia SK, Kalia LV and McLean PJ: Molecular chaperones as rational drug targets for Parkinson's disease therapeutics. CNS Neurol Disord Drug Targets 9: 741-753, 2010.

25. Harper SJ and LoGrasso P: Signalling for survival and death in neurones: The role of stress-activated kinases, JNK and p38. Cell Signal 13: 299-310, 2001.

26. Lamb JA, Ventura JJ, Hess P, Flavell RA and Davis RJ: JunD mediates survival signaling by the JNK signal transduction pathway. Mol Cell 11: 1479-1489, 2003.

27. Chan SH, Hsu KS, Huang CC, Wang LL, Ou CC and Chan JY: NADPH oxidase-derived superoxide anion mediates angiotensin II-induced pressor effect via activation of $\mathrm{p} 38$ mitogen-activated protein kinase in the rostral ventrolateral medulla. Circ Res 97: 772-780, 2005.

28. Shao Z, Bhattacharya K, Hsich E, Park L, Walters B, Germann U, Wang YM, Kyriakis J, Mohanlal R, Kuida K, et al: c-Jun $\mathrm{N}$-terminal kinases mediate reactivation of Akt and cardiomyocyte survival after hypoxic injury in vitro and in vivo. Circ Res 98: 111-118, 2006.

29. Kaelin WG Jr and Ratcliffe PJ: Oxygen sensing by metazoans: The central role of the HIF hydroxylase pathway. Mol Cell 30 393-402, 2008

30. Semenza GL: HIF-1 inhibitors for cancer therapy: From gene expression to drug discovery. Curr Pharm Des 15: 3839-3843, 2009.

31. Epstein AC, Gleadle JM, McNeill LA, Hewitson KS, O'Rourke J, Mole DR, Mukherji M, Metzen E, Wilson MI, Dhanda A, et al: C. elegans EGL-9 and mammalian homologs define a family of dioxygenases that regulate HIF by prolyl hydroxylation. Cell 107: 43-54, 2001.

32. Brennan J: Reperfusion injury of cardiac myocytes: Mechanisms, treatment, and implications for advanced practice nursing. AACN Clin Issues 11: 252-260, 2000.

33. Hausenloy DJ and Yellon DM: Myocardial ischemia-reperfusion injury: A neglected therapeutic target. J Clin Invest 123: 92-100, 2013.

34. Baines CP: How and when do myocytes die during ischemia and reperfusion: The late phase. J Cardiovasc Pharmacol Ther 16: 239-243, 2011

35. Braunwald E: The war against heart failure: The lancet lecture. Lancet 385: 812-824, 2015.

36. Zhao ZQ: Oxidative stress-elicited myocardial apoptosis during reperfusion. Curr Opin Pharmacol 4: 159-165, 2004.

37. Podsiad A, Standiford TJ, Ballinger MN, Eakin R, Park P, Kunkel SL, Moore BB and Bhan U: MicroRNA-155 regulates host immune response to postviral bacterial pneumonia via IL-23/IL-17 pathway. Am J Physiol Lung Cell Mol Physiol 310: L465-L475, 2016.

38. Bayraktar R and Van Roosbroeck K: miR-155 in cancer drug resistance and as target for miRNA-based therapeutics. Cancer Metastasis Rev 37: 33-44, 2018.
39. Gangwar RS, Rajagopalan S, Natarajan R and Deiuliis JA: Noncoding RNAs in cardiovascular disease: Pathological relevance and emerging role as biomarkers and therapeutics. Am J Hypertens 31: 150-165, 2018.

40. Zhai A, Qian J, Kao W, Li A, Li Y, He J, Zhang Q, Song W, Fu Y, $\mathrm{Wu}$ J, et al: Borna disease virus encoded phosphoprotein inhibits host innate immunity by regulating miR-155. Antiviral Res 98 : 66-75, 2013.

41. Cao S, Wang Y, Li J, Lv M, Niu H and Tian Y: Tumor-suppressive function of long noncoding RNA MALAT1 in glioma cells by suppressing miR-155 expression and activating FBXW7 function. Am J Cancer Res 6: 2561-2574, 2016.

42. Teramura $\mathrm{T}$ and Onodera Y: Stem cell depletion by inflammation-associated miR-155. Aging (Albany NY) 10: 17-18, 2018.

43. Tili E, Mezache L, Michaille JJ, Amann V, Williams J, Vandiver P, Quinonez M, Fadda P, Mikhail A and Nuovo G: microRNA 155 up regulation in the CNS is strongly correlated to Down's syndrome dementia. Ann Diagn Pathol 34: 103-109, 2018.

44. Bruchmann A, Roller C, Walther TV, Schafer G, Lehmusvaara S, Visakorpi T, Klocker H, Cato AC and Maddalo D: Bcl-2 associated athanogene 5 (Bag5) is overexpressed in prostate cancer and inhibits ER-stress induced apoptosis. BMC Cancer 13: 96, 2013.

45. Guo K, Li L, Yin G, Zi X and Liu L: Bag5 protects neuronal cells from amyloid $\beta$-induced cell death. J Mol Neurosci 55: 815-820, 2015.

46. Ying Z, Haiyan G and Haidong G: BAG5 regulates PTEN stability in MCF-7 cell line. BMB Rep 46: 490-494, 2013.

47. Yue X, Zhao Y, Huang G, Li J, Zhu J, Feng Z and Hu W: A novel mutant p53 binding partner BAG5 stabilizes mutant p53 and promotes mutant p53 GOFs in tumorigenesis. Cell Discov 2: $16039,2016$.

48. Kalia SK, Lee S, Smith PD, Liu L, Crocker SJ, Thorarinsdottir TE, Glover JR, Fon EA, Park DS and Lozano AM: BAG5 inhibits parkin and enhances dopaminergic neuron degeneration. Neuron 44: 931-945, 2004.

49. Bi L, Yang Q, Yuan J, Miao Q, Duan L, Li F and Wang S MicroRNA-127-3p acts as a tumor suppressor in epithelial ovarian cancer by regulating the BAG5 gene. Oncol Rep 36: 2563-2570, 2016

50. Bourke LT, McDonnell T, McCormick J, Pericleous C, Ripoll VM, Giles I, Rahman A, Stephanou A and Ioannou Y: Antiphospholipid antibodies enhance rat neonatal cardiomyocyte apoptosis in an in vitro hypoxia/reoxygenation injury model via p38 MAPK. Cell Death Dis 8: e2549, 2017.

51. Li Q, Xiang Y, Chen Y, Tang Y and Zhang Y: Ginsenoside Rg1 protects cardiomyocytes against hypoxia/reoxygenation injury via activation of Nrf2/HO-1 signaling and inhibition of JNK. Cell Physiol Biochem 44: 21-37, 2017.

(i)(9) This work is licensed under a Creative Commons Attribution-NonCommercial-NoDerivatives 4.0 International (CC BY-NC-ND 4.0) License. 